\title{
Germanica
}

\section{Le poids de la mémoire. Quelques réflexions sur la nouvelle Tonka de Robert Musil}

Die Last des Gedächtnisses. Nachdenten über Robert Musils Novelle Tonka

\section{Claus ERHART}

\section{OpenEdition}

\section{Journals}

Édition électronique

URL : http://journals.openedition.org/germanica/1823

DOI : 10.4000/germanica. 1823

ISSN : 2107-0784

\section{Éditeur}

Université de Lille

\section{Édition imprimée}

Date de publication : 31 décembre 2003

Pagination : 57-77

ISBN : 9782913857124

ISSN : 0984-2632

\section{Référence électronique}

Claus ERHART, «Le poids de la mémoire. Quelques réflexions sur la nouvelle Tonka de Robert Musil », Germanica [En ligne], 33 | 2003, mis en ligne le 30 novembre 2012, consulté le 06 octobre 2020. URL: http://journals.openedition.org/germanica/1823 ; DOI : https://doi.org/10.4000/germanica.1823

Ce document a été généré automatiquement le 6 octobre 2020.

(C) Tous droits réservés 


\title{
Le poids de la mémoire. Quelques réflexions sur la nouvelle Tonka de Robert Musil
}

\author{
Die Last des Gedächtnisses. Nachdenten über Robert Musils Novelle Tonka
}

\section{Claus ERHART}

1 En 1902, après avoir terminé ses études de construction mécanique et son service militaire, Robert Musil quitte Brünn, où sa famille réside depuis 1891. Il s'installe pour une année à Stuttgart et y travaille comme assistant du célèbre professeur Carl Bach, à l'école polytechnique. Insatisfait de son métier, Musil se met à écrire. Dans le texte Testament II (Vermächtnis II) de 1932, il se souvient :

Chaque soir, vers $9 \mathrm{~h} 1 / 2$, une amie venait me voir, mais je rentrais déjà du bureau à 6 heures. Stuttgart, où ces choses se passaient, m'était étranger et hostile, je voulais abandonner mon métier et étudier la philosophie (ce que je fis bientôt), je fuyais mon travail, je lisais des ouvrages philosophiques pendant mes heures de bureau et à la fin de l'après-midi, quand je ne me sentais plus capable de rien enregistrer, je m'ennuyais. C'est ainsi que je commençai à écrire, et le thème que j'avais sous la main se trouva être justement celui de Törless ${ }^{1}$.

2 Cette « amie » qui venait régulièrement voir le jeune homme est probablement Herma Dietz ${ }^{2}$. Elle servira de modèle pour le personnage de Tonka dans la nouvelle du même nom, qui paraîtra la première fois en 1922/23, puis en 1924 dans le recueil Drei Frauen.

Herma Dietz a été pendant six années environ la compagne de Robert Musil, mais on ne sait que peu de choses sur elle. Fille du peuple, elle travaille dans un magasin de tissus à Brünn ${ }^{3}$ lorsqu'elle rencontre en 1901 celui qu'elle suivra d'abord à Stuttgart, puis à Berlin. Leur relation entre en crise lorsque Herma, en 1905/06, tombe enceinte et développe les symptômes de la syphilis ${ }^{4}$. Elle meurt en 1907, probablement seule, des suites d'un avortement syphilitique et, comme l'écrit Karl Corino, de la cruauté mentale de son $\mathrm{ami}^{5}$.

4 Dans son journal, Musil ne lui accorde pas beaucoup de place, ni beaucoup d'estime. Alors que la passion pour « Valerie » (l'actrice Paula Ullmann) avait flatté l'ego du jeune 
esthète - il se voit, par la force de ses sentiments, élevé au rang des grandes incarnations de l'amour tel Jésus Christ, Bouddha ou encore Goethe (cf. Tb I, 12) - la simplicité de Herma ne provoque pas chez lui de grands élans amoureux. Nous apprenons dans son journal qu'il l'a déflorée, il y parle d'une promenade en traîneau (cf. $\mathrm{Tb} \mathrm{I}, 11)$ et dans une conversation fictive, il lui avoue qu'il ne la trouve pas à son niveau ${ }^{6}$. Et très vite, elle perd dans les notes de son ami son identité propre pour se fondre avec d'autres noms dans les ébauches, dans les réflexions fragmentaires sur des sujets à exploiter par « monsieur l'écrivain »?

5 Après les impressions reçues au lycée militaire de Mährisch-Weisskirchen, qui ont fourni des matériaux pour Les désarrois de l'élève Törless, c'est donc au tour de Herma Dietz et à leur relation de devenir «le thème " que l'auteur aura un jour "sous la main $»^{8}$. Si l'on ne sait pas exactement quand Musil est passé des esquisses vagues à la concrétisation littéraire de ce sujet, il existe néanmoins deux lettres, datées du mois d'octobre 1922, où il est question de l'achèvement imminent de la nouvelle Tonka (cf. B I, p. 272, 274 s.).

6 L'auteur Musil a souvent manifesté son indifférence pour le côté événementiel d'un sujet. Dans une ébauche d'essai (Profil eines Programmes [1912]), on peut par exemple lire que

les événements extérieurs ainsi que les phénomènes psychologiques ne sont, en réalité, que la part impersonnelle du destin. (GW II, 1320 [trad. C.E.])

7 La dévalorisation de l'action est un élément constitutif de sa théorie artistique. Ainsi, dans les textes critiques que Musil a rédigés en tant que chroniqueur dramatique, la description détaillée de l'intrigue implique le plus souvent une critique mordante de la pièce ${ }^{9}$. Quant à ses propres ambitions, on lit dans l'essai Der Schwärmerskandal de 1929 :

Ce qui m'importe en réalité, ce n'est pas ce que je raconte ni la personne que je décris. Je ne veux qu'une chose : leur insuffler le plus possible de vie de l'esprit. (GW II, 1192 [trad. C.E.])

$8 \quad$ Et dans un article sur le théâtre d'art de Moscou paru dans la Prager Presse du 21 avril 1921, il explique qu' « un écrivain authentique [...] donne forme », dans la moindre de ses trouvailles, «à sa vision du monde » (cf. E, 446 ; GW II, 1479).

9 À partir de ces quelques réflexions à caractère programmatique, on peut s'interroger sur les raisons qui ont amené Musil à reprendre un sujet qu'il a gardé sous le coude pendant au moins quinze ans. De quelles idées a-t-il pu enrichir son vécu avec Herma Dietz? Quelle «vie de l'esprit » a-t-il « insufflé » à leur misérable histoire ? Est-ce que Tonka est porteuse de sa vision du monde, et l'est-elle au même titre que Grigia et $L a$ Portugaise, les deux autres nouvelles du recueil Trois femmes, paru en 1924 ?

10 Arrêtons-nous un instant sur la pensée de Robert Musil à l'époque où on peut supposer que le projet de Tonka commence à se concrétiser. Selon Hermann Broch, lui-même incontestablement partisan de la clarté, Musil « est peut-être l'écrivain le plus exact que la littérature mondiale ait jamais produit $»^{10}$. Si l'exactitude représente pour Musil une vertu majeure, il y a là sans doute un rapport avec sa formation, peu typique d'un écrivain de sa génération. Après les études d'ingénieur et l'année d'assistanat à l'école polytechnique de Stuttgart, Musil s'inscrit à l'université de Berlin, où il suit des cours de philosophie (il s'intéresse tout particulièrement à la logique), de psychologie, de mathématiques et de physique. En 1908, il soutient une thèse de doctorat sur l'épistémologie d'Ernst Mach, mais renonce par la suite à une carrière universitaire pour devenir écrivain. Dans ce choix de la littérature se profile déjà une particularité de 
l'esthétique musilienne, puisqu'il semble être motivé par les lacunes fondamentales que l'auteur a découvertes dans la démarche scientifique et qui ont déjà troublé le jeune Törless ${ }^{11}$.

11 Les limites de la science et de la raison sont un sujet récurrent dans l'œuvre de Musil. Ainsi, on lit par exemple dans l'essai Confession politique d'un jeune homme de 1913 que la pensée scientifique « aligne les points avec la limpidité d'une machine à coudre », mais qu'elle nous « appauvrit » puisqu'elle ne nous accorde « de réponses qu'à une ou deux questions dont » notre « âme [...] ne peut vivre » $(\mathrm{E}, 61 \mathrm{~s} . ;$ GW II, 1009) et dans la pièce Les exaltés, Thomas, un savant renommé, s'adresse à Anselm, son confrère, en s'exclamant :

Qu'avons-nous obtenu ? Dans notre cabinet de travail, nous avons réfléchi comme le singe qui, un caillou à la main, se demande comment il peut ouvrir la noix. Sans parvenir à répondre à la moindre des questions qui touchent à notre félicité d'hommes. (GW II, 331 [trad. C.E.])

12 Ces citations pourraient laisser penser que Musil rejoint là la pensée d'un Oswald Spengler, d'un Walther Rathenau, d'un Ludwig Klages et d'autres « beaux esprits » (cf. E, 112; GW II, 1055), qui ont joué un rôle important dans la vie culturelle du début du $\mathrm{xx}^{\mathrm{e}}$ siècle. En réalité, il a toujours gardé ses distances vis-à-vis des prédicateurs de l'intuition, comme on le voit entre autres dans L'homme sans qualités, où il brosse leur portrait ironique. Dans le grand roman, ils fréquentent le salon de la divine Diotime, où Ulrich reste un élément étranger car il « les gêne » avec son " sérieux » (L'Hsq, $577 ; \mathrm{GW}$ I, 459). Ce sérieux est sensiblement le même que celui que Jacques Bouveresse constate chez Musil, celui de l'homme exact face aux avancées de l'à-peu-près :

Ce que Musil reproche aux écrivains et aux artistes de son époque n'est évidemment pas de se poser en défenseurs de l'âme, mais de n'avoir généralement pas trouvé d'autre moyen de le faire que celui qui consiste à déclarer la guerre à l'intellect, sans être capable de lui opposer autre chose que des notions et des proclamations dont la maigreur et l'enflure ont toutes les chances de constituer précisément le meilleur argument en sa faveur [.] ${ }^{12}$

Musil n'est pas le seul à condamner cette déclaration de guerre à l'intellect. Max Weber, dans une conférence de novembre 1917 (Wissenschaft als Beruf, parue en 1919), déplore la position extrêmement négative prise par beaucoup de ses contemporains contre le processus de l'intellectualisation ${ }^{13}$, et il fustige le besoin répandu de se délivrer du rationalisme par l'expérience ${ }^{14}$. Mais de tels appels restent sans écho, dans cette république de Weimar qui marque, dans l'histoire des idées, un aiguillage vers l'irrationalisme ${ }^{15}$. Parmi les nombreux faits révélateurs de cette orientation, on peut citer au moins un exemple : les cérémonies officielles à l'occasion du $200^{\mathrm{e}}$ anniversaire de Gotthold Ephraim Lessing en 1929. Lessing est le premier auteur de renommée internationale dont la jeune république doit célébrer la mémoire. À cette occasion, peu d'orateurs peuvent se résoudre à apprécier sans réserve son programme rationaliste et l'on s'efforçe plutôt de voir en lui non pas un des principaux représentants de l' Aufklärung, mais celui qui a su la dépasser ${ }^{16}$. Et même Thomas Mann, qui admire par ailleurs la clarté intellectuelle de l'auteur, qualifie l'Aufklärung chez Lessing d'« intellectuellement désuète » et affirme qu'« elle a fait place, à l'heure actuelle, à une conception plus charnue, plus profonde et plus tragique de la vie $»^{17}$. En évoquant ici, de manière emphatique, une certaine " conception de la vie ", Thomas Mann se réfère à la Lebensphilosophie, que Robert Musil, quant à lui, en dépit de quelques convergences, ne cesse de critiquer $^{18}$. Cette dernière oppose, comme il le démontre dans son essai sur 
Le Déclin de l'Occident d'Oswald Spengler, l'intuition à l'intellect, l'âme à l'analyse, et fournit ainsi à la Gegenaufklärung de son époque quelques-uns de ses plus séduisants arguments.

Quand Musil regarde rétrospectivement l'évolution de l'esprit moderne, il considère l' Aufklärung comme la dernière époque marquée par un bel optimisme, dans la mesure où

les hommes de ce xviiie siècle finissant croyaient à quelque chose en nous qui n'attendait que d'être libéré pour jaillir vers le Ciel. Ils l'avaient baptisé la Raison [...]; ils n'avaient pour la tradition qu'un respect limité et se faisaient fort [" trauten sich zu »] de rebâtir le monde à partir de l'esprit. (E, 142 ; GW II, 1082)

Cette pensée avait «la violence contagieuse d'une expérience nouvelle et libératrice » (E, 145 ; GW II, 1085), mais la " construction ", à laquelle elle aboutissait, "s'écroula ", puisqu'elle reposait sur des bases intellectuelles beaucoup trop étroites (cf. E, 142 ; GW II, 1082), ce qui est, selon Musil, l'héritage de la Renaissance. D'après lui, à cette époque s'est produite une "restitutio in integrum", au cours de laquelle la rationalité, dégradée par la "spéculation scolastique ", a trouvé «tel Antée, le terrain solide des faits » (E, 145 ; GW II, 1084). Elle était le point de départ de deux évolutions : d'un coté, l'essor du rationnel qui mène, en passant par l'Aufklärung, au positivisme du XIX siècle, de l'autre, en liaison avec les progrès de l'intellect, un besoin croissant d'irrationnel, « la protestation du sentiment, de la volonté, du muable [!] [« des Wechselbaren »] et du vivant » (E, 154 ; GW II, 1092).

Le chaos qui règne dans le domaine de l'esprit au début du $\mathrm{xx}^{\mathrm{e}}$ siècle est dû pour Musil au fait qu'on n'ait jamais accordé le rationnel et l'irrationnel dans un ensemble synthétique, mais qu'on ait conservé leur antagonisme au lieu de le résoudre « en des séries de transitions » (cf. E, 148 s.; GW II, 1087 s.). Enfermer les deux aspects de l'esprit humain dans une opposition rigide est intellectuellement insatisfaisant, puisque ni le rationnel ni une pensée se définissant par le refus de ce dernier ne peuvent apporter de réponses aux problèmes qui se posent dans l'« Europe désemparée $»^{19}$ :

Les concepts qui pourraient ordonner la vie qui nous entoure font défaut. Les faits du passé, les faits des diverses sciences et les faits de la vie nous submergent pêlemêle. Quand la philosophie vulgarisée [«Populärphilosophie»] et les discussions quotidiennes [«die Tagesdiskussion»] ne se contentaient pas des défroques du libéralisme avec sa croyance aveugle en la raison et le progrès, elles inventaient ces trop fameuses idoles ["Fetische»] que sont l'époque, la nation, la race, le catholicisme, l'homme intuitif; toutes ayant pour dénominateur commun négatif une hargne sentimentale à l'égard de l'intellect et, en positif, le besoin d'un étai [«Halt»], de grands squelettes fantômes où accrocher les impressions à quoi l'on s'était laissé réduire. (E, 148 ; GW II, 1087)

Dans la littérature aussi, Musil trouve le reflet de cet antagonisme stérile. Alors que le naturalisme a "produit de la réalité à laquelle manquait l'esprit ", l'expressionnisme, lui, a fait « de l'esprit auquel manquait la réalité ; l'un comme l'autre, du «non-esprit » [« Ungeist »]» (E, 116; GW II, 1058 s.). Avec les expressionnistes, Musil partage l'envie de voir apparaitre un homme nouveau, mais il critique leur manière de penser « en termes vides auxquels faisaient défaut le contenu, et le contrôle de l'expérience » (ibid.).

18 L'entrée « dans une ère nouvelle » $(\mathrm{E}, 63$; GW II, 1011), le développement de " quelque mode encore inconnu d'être un homme » (E, 38 ; GW II, 989) passent pour Musil par une synthèse. Il s'agit de remplacer la pensée rationnelle par une pensée « émo-rationnelle 
[ « emotio-rational »] et senti-mentale » (E, $61 ;$ GW II, 1008), qui ne soit pas l'opposée de la première, mais la complète en lui associant des modes de perception qui s'appliquent dans le « domaine non ratioïde ». L'auteur s'excuse de la « laideur du néologisme » (E, 81 ; GW II, 1026) et tente une définition:

Si le domaine ratioïde était celui de la règle avec exceptions, le domaine non ratioïde est celui où les exceptions l'emportent sur la règle. Peut-être n'y a-t-il là qu'une différence de degré ; mais, quoi qu'il en soit, si capitale qu'elle nécessite un renversement complet de la position du sujet connaissant ["vollkommene Umkehrung der Einstellung des Erkennenden »]. Dans ce domaine, les faits ne sont pas dociles, les lois sont des cribles, les événements ne se répètent pas, mais sont infiniment variables et individuels. Je ne puis mieux le caractériser qu'en précisant qu'il est le domaine des réactions ["Reaktivität»] de l'individu au monde et à autrui, le domaine des valeurs et des évaluations, des relations éthiques et esthétiques, le domaine de l'idée. (E, 82 s. ; GW II, 1028)

L'importance de ces idées pour l'œuvre de Musil est capitale. Avec la synthèse de «la pensée scientifique » et des «exigences de l'âme» (E, 116 s.; GW II, 1059), l'auteur a trouvé le sujet de sa vie, dont on voit les premières traces distinctes dans sa théorie artistique.

Si après avoir passé son doctorat, Musil a quitté l'univers scientifique, il n'est pas prêt pour autant à sombrer dans l'irrationalisme. Il ne renonce pas à la connaissance, mais cherche plutôt à l'étendre à d'autres domaines comme celui de l'art. Ce qui distingue l'art de la science, c'est qu'il

représente [...] non pas le général, mais des cas particuliers dont la sonorité complexe englobe aussi de vagues notes générales; et tandis que, confronté au même cas, le medecin s'intéresse à une relation causale de valeur générale, l'artiste s'intéresse à une relation affective individuelle [...]. (E, 29 ; GW II, 980 s.)

Alors que

la science n'a d'instruments ["Organ»] et d'intêrét que pour ce qui se reproduit chroniquement [« für das Wiederkehrende im Wechsel»], jamais pour l'événement unique ou isolé (E, 40 ; GW II, 990),

la tâche de l'écrivain consiste, selon Musil, à

surprendre des connaissances affectives [ «Gefühlserkenntnisse »] et des ébranlements intellectuels que l'on ne peut saisir ni de façon générale ni conceptuellement, mais uniquement dans le papillotement des cas particuliers [«im Flimmern des Einzelfalls»]; ou peut-être, autrement dit: que l'on ne peut saisir dans cette totalité qu'est l'homme rationnel, bourgeoisement doué pour les affaires, seulement dans des éléments moins solides, mais qui le dépassent. (E, 48 ; GW II, 997)

23 On voit bien le lien entre ces idées, formulées de 1911 à 1913 dans les premiers essais, et le personnage de Tonka tel qu'il apparaît environ dix années plus tard dans la nouvelle. La jeune femme incarne si bien le "papillotement des cas particuliers ", elle représente un cas tellement "unique » et "isolé " que l'auteur, pour la "saisir», a recours à l'image audacieuse $d^{\prime}$ « un flocon de neige qui tomberait en plein été » (Tf, 123 $)^{20}$.

24 Ainsi, dans la poétique que Musil développe, de manière systématique à partir de 1910, la littérature, non seulement

présuppose la connaissance, mais elle la prolongue [...] jusque dans cette région frontalière du pressentiment, du plurivoque et du singulier où l'on ne peut aborder avec les seuls moyens de l'entendement. (E, 331 ; GW II, 1327) 
Sittérature veut acquérir des connaissances au-delà des bornes de l'entendement, elle ne peut pourtant pas faire cavalier seul. Elle doit, au contraire, prendre modèle sur l'« intelligence scientifique», s'inspirer de son "absence de préjugés» et de sa "volonté de remettre chaque résultat en question». La littérature doit, en un mot, «faire dans les problèmes de la vie » (« in den Fragen des Lebens») ce que fait la science dans son domaine (cf. E, 66; GW II, 1011). Pour souligner l'exemplarité de la pensée rationnelle, Musil emploie parfois des formulations provoquantes, comme dans Les exaltés où il fait dire à Thomas :

Celui qui ne sait résoudre une intégrale ou qui ne maitrise pas la technique expérimentale ne devrait pas être autorisé, aujourd'hui, à se prononcer sur les problèmes de l'âme. (GW II, 392 [trad. C.E.])

Avec de pareils propos, Musil fait clairement comprendre que le rationalisme, à ses yeux, n'est pas caduc et que la précision et la lucidité de l'intellect restent des moyens indispensables dans l'approche du «non ratioïde ». Les " problèmes de l'âme » n'étant pas solubles par la seule raison, il ne peut pourtant pas s'agir de faire abstraction de celle-ci et de remplacer la connaissance scientifique par de prétendues « connaissances affectives » [«Gefühlserkenntnisse »] (E, 48; GW II, 997). Les mots de Thomas rappellent ceux d'Ulrich, l'homme sans qualités, qui, penché sur des confessions de mystiques et désireux de freiner l'enthousiasme de sa sœur pour leurs visions, veut examiner «aussi froidement que possible ce qui se produit là » (L'Hsq 2, 106; GW I, 753), car il

était convaincu qu'on ne pouvait rien gagner à céder à des imaginations qui ne résistaient pas à un examen attentif. C'était comme les ailes de cire d'Icare qui fondirent à l'altitude, s'écria-t-il; si on ne voulait pas voler seulement en rêve, il fallait apprendre à le faire avec des ailes de métal. (L'Hsq 2, 122 ; GW I, 765 s.)

'attitude que l'homme sans qualités démontre ici est la prudence de l'esprit scientifique qui s'apprête à explorer un domaine fascinant et jusqu'alors réputé inexact. La plupart des personnages principaux de Musil, à commencer par Törless, ont en commun cet émerveillement devant un terrain inconnu. En 1920, dans un de ses carnets, l'auteur appelle la «rationalité » et la «mystique » les deux "pôles » de son époque (cf. Tb I, 389). Ils correspondent aux forces de l'esprit humain, que l'écrivain et la littérature devraient concilier en une synthèse. Désormais, les protagonistes masculins - Thomas et Ulrich aussi bien que Homo, le seigneur von Ketten et l'ami de Tonka dans les Trois femmes - seront tous, chacun à sa manière, marqués par le rationnel. Tous se rendront compte, à un moment de leur vie, de l'étroitesse de leur vision du monde et ils réagiront en s'ouvrant à une expérience nouvelle, tous, à l'exception de $\mathrm{N}^{21}$, le personnage principal de la nouvelle Tonka.

Dans les Trois femmes, le deuxième pôle, la «mystique » ou - plus généralement - le " non ratioïde », est incarné par les héroïnes. Tonka, elle, qui

était si simple et si transparente que l'on pouvait se croire le jouet d'une hallucination ou d'un mirage (Tf, 82), (Tb I, 185).

La jeune femme est employée dans un magasin de tissus, quand elle fait la connaissance de $\mathrm{N}$, qui la fait embaucher par sa famille pour soigner la grand-mère malade et lui tenir compagnie. Après la mort de la vieille dame, $\mathrm{N}$ propose à Tonka de s'installer avec lui dans une grande ville allemande où il travaille à une invention dans le domaine de la 
chimie. Après quelques années de vie commune, Tonka s'aperçoit qu'elle est enceinte et à partir de ce moment tout n'est "plus qu'épineuses broussailles " («war alles in ein Dornengerank verwandelt worden » [Tf, 97 ; GW II, 288)]. Car en faisant le calcul, N doit constater que la date de la conception correspond à une période d'absence. Et puis Tonka tombe malade. Il s'agit d'" une maladie effrayante, dangereuse, sournoise", d'une maladie qui peut être transmise "au sang de la mère soit par l'enfant, soit, directement, par le père " (Tf, 98). Comme $\mathrm{N}$, selon toute apparence, est en bonne santé, il conclut qu'il est confronté ou bien à l'infidélité de sa compagne ou bien à un phénomène surnature ${ }^{22}$. Tonka succombe à sa maladie avant l'accouchement, au moment même où $\mathrm{N}$ a achevé son invention.

Par tout son être, Tonka évoque un monde à part, au-delà de ce qui est à la portée de la pensée ${ }^{23}$, et ainsi que Musil note dans son journal, la malédiction pesant sur elle et son ami met symboliquement en évidence que, dans un sens, on ne peut absolument pas se fier à la raison (cf. Tb I, 100).

L'idée que Tonka n'est pas un personnage comme les autres, avec une identité déterminée, mais qu'elle est, avant tout, comme le dit Ulrich dans le grand roman à propos de notre monde, « une image, une analogie » (L'Hsq 1, 449; GW I, 357) est omniprésente dans la nouvelle. Dans les premières phrases, on a l'impression d'assister à la naissance ou à la recomposition de la jeune femme dans la mémoire du narrateur :

Une barrière. Le chant d'un oiseau. Le soleil déjà disparu derrière les buissons. Le chant qui se tait, le soir qui vient. Les chanson des jeunes paysannes à travers champs. De biens petits détails! Que de tels détails s'accrochent à quelqu'un comme des bardanes, faut-il le croire mesquin ? C'était cela, Tonka. L'infini vous est donné parfois au compte-gouttes. (Tf, 69 ; GW II, 270)

Et un peu plus loin :

[...] il l'avait aperçue d'abord appuyée à une barrière, devant une sombre porte béante, dans la première maison du village en allant vers la ville. [...] elle se sentait protégée par la lune, et le vent soufflait sur les éteules aussi doucement que s'il avait eu une soupe [...] à refroidir. (Tf, $72 ; \mathrm{GW}$ II, 272)

Tonka, tel que le lecteur l'aperçoit ici, est plongée dans une atmosphère, qui est comparable à celle créée par le Gleichnis, dont le sens, comme le décrit Musil, ne se dévoile que pendant de brefs instants ${ }^{24}$. Dans le grand roman, Ulrich reconnaitra que la métaphore («Gleichnis »), profondément liée au « non ratioïde », ne peut être ramenée à une vérité univoque :

Mais quand, dans une métaphore [«Gleichnis»], on dissocie tout ce qui pourrait être vrai de ce qui n'est qu'écume, on ne fait d'ordinaire que gagner un peu de vérité en détruisant toute la valeur de la métaphore. Cette dissociation peut avoir été inévitable dans l'évolution intellectuelle, mais elle a eu le même effet que lorsqu'on met bouillir une substance pour l'épaissir: l'évaporation, en cours d'opération, du meilleur d'elle-même. (L'Hsq 1, 747 ; GW I, 593)

Tonka se dérobe à cet «épaississement » et l'énigme de son identité traverse toute la nouvelle. $\mathrm{N}$ se doute qu'elle continue, « au-dessous de tout ce que le monde lui » fait, «à être quelque chose de plus profond " (Tf, 123; GW II, 304), mais il n'arrive pas à comprendre de quoi il s'agit. Son travail avance, son "personnage social» ("bürgerliche Person») s'affirme, mais " au moindre rappel en lui de l'existence de Tonka, des personnages" se mettent "à tourner dans sa tête sans révéler leur signification » (Tf, 115 ; GW II, 299). C'est comme si l'être humain qu'est la jeune femme s'effaçait devant sa valeur de signe; mais ce signe est équivoque et ne se laisse pas 
traduire dans un langage clair et distinct. Et puis une idée effleure l'esprit de N, l'idée que « connaître Tonka » veut dire « être obligé de lui [...] crier [« ihr entgegenrufen »] qui elle » est (cf. Tf, 111 ; GW II, 296).

Il y a là un changement de point de vue. Dans cette pensée de son ami, Tonka n'est plus un signe qui renvoie à une réalité secrète et qui demande à être lu et compris, mais elle est plutôt quelque chose d'indéfinissable et de fugace que l'on essaie de maîtriser en l'accrochant à un sens. Musil désapprouve un tel usage usurpatoire de la raison ${ }^{25}$, où celle-ci ne s'adapte pas au domaine "non ratioïde ", mais opère comme un principe hostile, où

on enfonce dans la profondeur indéterminée les pilotis des concepts [«die erstarrenden Caissons der Begriffe »] autour desquels s'établit tout un réseau de lois, de règles et de formules. (E, 82 ; GW II, 1027)

Dans Histoire de la folie à l'âge classique, Michel Foucault étudie les mécanismes qu'utilise la rationalité pour s'assurer l'hégémonie, et plus particulièrement la domination qu'elle commence à exercer sur la folie et d'autres comportements suspects au seuil du XVII ${ }^{\mathrm{e}}$ siècle. Au Moyen Âge et encore à l'époque de la Renaissance, la société imposait souvent aux aliénés une existence errante. On les chassait des villes, soit en les laissant "courir dans les campagnes éloignées", soit en les confiant à des marchands itinérants, à des pèlerins ou encore à des bateliers ${ }^{26}$. L'embarquement des fous, notamment, est pour Foucault un acte hautement symbolique :

C'est vers l'autre monde que part le fou sur sa folle nacelle; c'est de l'autre monde qu'il vient quand il débarque. Cette navigation du fou, c'est à la fois le partage rigoureux, et l'absolu Passage ${ }^{27}$.

À l'âge classique, la notion de passage (d'un monde à un autre, d'une "science déréglée et inutile $»^{28}$ à un " savoir difficile, fermé, ésotérique $»^{29}$ ) disparaît alors que le partage persiste mais sous une nouvelle forme. C'est alors que la raison prend une dimension despotique, en reprenant, groupant et bannissant «certaines expériences » que le $\mathrm{xVI}^{\mathrm{e}}$ siècle encore "avait acceptées ou [...] laissées en marge », et en les envoyant "dans l'exil où elles voisineront avec la folie - formant ainsi un monde uniforme de la Déraison ». Parmi les « expériences » qui subissent le même sort que la folie, à savoir internement et châtiment (corporel), figurent en premier lieu toutes celles qui "touchent [...] à la sexualité dans ses rapports avec l'organisation de la famille bourgeoise $\aleph^{30}$, comme la prostitution et la débauche, et en particulier si elles deviennent visibles en prenant l'aspect du mal vénérien. Ainsi, l'époque classique trouve «à la fois une patrie et un lieu de rédemption communs aux péchés contre la chair et aux fautes contre la raison $»^{31}$. Dans ce contexte d'oppression la famille bourgeoise joue un rôle décisif. C'est elle qui «trace le cercle de la raison » et elle en réduit le rayon afin d'exclure « comme étant de l'ordre de la déraison » tout ce qui va à l'encontre de son intérêt: "Débauche, prodigalité, liaison inavouable, mariage honteux » sont des motifs « classiques » de l'internement ${ }^{32}$.

39 Sous cette emprise, la «folie devient une forme relative à la raison $»^{33}$, elle devient "l'Autre de la raison selon le discours de la raison même " ${ }^{34}$, car la folie, elle, appartient au XVII ${ }^{e}$ siècle " aux régions du silence $»^{35}$. Il existe des textes qui parlent d'elle, des livres dans lesquels

on la prend de biais, dans sa dimension négative, parce qu'elle est une preuve «a contrario » de ce qu'est, dans sa nature positive, la raison. [...] En elle-même, elle est chose muette[.] ${ }^{36}$ 
40 À propos de la représentation désormais obsolète d'une folie circulante, on lit chez Foucault :

L'oubli tombe sur le monde que sillonnait le libre esclavage de sa Nef : elle n'ira plus d'un en-deçà du monde à un au-delà, dans son étrange passage ; elle ne sera plus jamais cette fuyante et absolue limite. La voilà amarrée, solidement, au milieu des choses et des gens. Retenue et maintenue. Non plus barque mais hôpital ${ }^{37}$.

41 Il y a dans tout cela des similitudes manifestes avec la nouvelle de Musil ; l'histoire de Tonka est comme une adaptation moderne du destin de la déraison au seuil de l'âge classique. Par son nom de famille, « un de ces fabuleux noms tchèques qui signifient « Il chantait » ou encore " Il est venu à travers prés » » (Tf, 83 ; GW II, 279), Tonka semble avoir des liens de descendance avec les fous errants d'une ère précédant l'intronisation du rationalisme. Dans une ébauche, Musil établit également un rapport entre son héroïne et ce lointain passé en la comparant à un tableau du gothique $\operatorname{tardif}^{38}$, et dans la nouvelle il souligne le décalage entre Tonka et son temps de façon explicite :

[...] peut-être qu'en une autre époque Tonka aurait été une femme illustre dont les princes mêmes [n'] [ !] auraient hésité à demander la main : mais de nos jours ? (Tf, 122 ; GW II, 304)

42 Tonka évolue dans une zone indéterminée - elle «n'était pas bête, mais quelque chose semblait l'empêcher d'être intelligente » (Tf, 75 ; GW II, 274) -, ce qui la rend vulnérable face aux exigences d'un monde rationnel et pragmatique. Elle ne peut se défendre car, à l'instar de la folie dont parle Foucault, elle ne maîtrise pas la parole : «Comme Tonka était muette !» (Tf, 85); du moins est-elle incapable, comme le dit Renate Homann, de faire prévaloir sa volonté, ses désirs et ses intérêts ${ }^{39}$. Le jour où elle est congédiée, sa faiblesse est évidente :

Elle ne put prononcer un mot, mais ses yeux s'emplirent de larmes. Qu'elle ne pût parler, l'homme raisonnable ne s'en émut guère [.] (Tf, 105 ; GW II, 293)

Et dans un premier moment 0se avec $\mathrm{N}$ :

Il lui caressa la main.

- Je crois que nous nous entendons bien, Tonka, mais me comprenez-vous?

Au bout d'un moment, elle répondit :

- Peu importe que je sache ou non ce que vous voulez dire. De toute façon je ne pourrais pas répondre. Mais j'aime que vous soyez si sérieux. (Tf, 81 s. ; GW II, 278)

Le fait d'être muette condamne Tonka à subir le discours des autres. Comme elle perturbe l'ordre rationnel des choses, ce discours se veut, conformément aux mécanismes démontrés par Foucault, discriminatoire et disciplinant.

Tonka trouble l'ordre de deux manières différentes. D'une part les membres de la famille de $\mathrm{N}$ la considèrent comme une intruse, puisqu'elle ne correspond pas à leurs critères sociaux: "Mais ce n'est qu'une fille tout à fait simple, une employée du magasin de tissus " (Tf, 88; GW II, 282) disent-ils, quand N part vivre avec elle en Allemagne. Ils voient en elle

une sorte d'oiseau de malheur [« ein böses Zeichen »], puisque c'était par elle que le cours habituel de leur vie avait été pour la première fois troublé. (Tf, 102 ; GW II, 291)

Lorsque Tonka tombe enceinte et que le danger d'une mésalliance devient imminent, la famille est bien décidée à faire cesser le désordre. Le discours devient alors plus rigoureux et Tonka est accusée de débauche et de sorcellerie érotique. Elle est qualifiée de « fille perdue », faisant « fi du bonheur d'une famille » et la mère ne manque pas de faire de « maladroites allusions [...] aux « artifices amoureux » grâce auxquels » la jeune 
femme aurait « envoûté » son fils (cf. Tf, 102 s.; GW II, 291). Même si ce dernier prend parti pour Tonka, les sous-entendus perfides de sa mère produisent leur effet ségrégatif. Plus tard encore, quand $\mathrm{N}$ s'efforce de reconstituer l'enfance de sa compagne décédée, où elle a cotoyé des prostituées, comme la cousine Julie, ou d'autres détenues d'une maison d'arrêt proche, il s'interroge sur l'absence de honte chez la jeune Tonka :

On jugera peut-être que c'était de l'imprudence de livrer une jeune créature sans défense à des influences propres à la blaser [« Ahnungslos mag man das nennen, ahnungslos ausgeliefert sein eines jungen, armen Lebens an Einflüsse, die es abstumpfen müssen »]; mais quand plus tard, à seize ans, Tonka continua de plaisanter tranquillement avec la cousine Julie, peut-on dire qu'elle fût encore inconsciente de la dépravation, ou son âme n'y était-elle déjà plus sensible du tout? Même si ce n'était pas sa faute, ce trait ne serait-il pas extrêmement révélateur? (Tf, $71 ;$ GW II, 271)

Le doute sur la sincérité et la moralité de Tonka s'est alors installé dans l'esprit de $\mathrm{N}$ et ne l'a plus jamais quitté.

Par ailleurs, Tonka trouble l'ordre en niant toute infidélité, donc en défiant l'autorité du rationnel même. Si l'on admet ses affirmations, sa grossesse et sa maladie relèvent du miracle et elle même rejoint alors « le pays profond des légendes [ "Märchen »], le pays de l'Oint du Seigneur, de la Vierge et de Pilate » (Tf, 100; GW II, 289). Le thème principal de la nouvelle n'est donc pas le problème de la jalousie, mais le problème de la véritét $^{00}$, et il risque d'ébranler sur ses bases la pensée scientifique de N. Devant cette menace, il emploie un discours qui reproduit le partage raison/déraison, notamment dans sa dimension discriminatoire.

Dès le début, l'attitude de $\mathrm{N}$ vis-à-vis de Tonka est ambiguë, ce qui semble refléter l'incertitude qui le guette. Après la mort de la grand-mère, il propose à Tonka de venir vivre avec lui, mais lui parle aussi des «femmes supérieures » qu'il connaîtrait plus tard (cf. Tf, 87 s. ; GW II, 281 s.). Convaincu de son infidélité, il ne veut plus dormir avec elle (cf. Tf, 102; GW II, 290), mais reste aux côtés « de sa compagne enceinte dont rien n'eût pu le séparer » (cf. Tf, 106; GW II, 293). Il a toujours éprouvé " pour elle cette immense compassion [...] si difficile à motiver » (Tf, 76 ; GW II, 274), mais quand il veut, quelque temps après, que la jeune femme lui "appartienne entièrement", il lui fixe " une date, comme un huissier " (Tf, 95 ; GW II, 286). Et le jour venu, comme Tonka s'efforce de dissimuler son émotion, il croit

voir sa propre pensée s'enrouler autour des chevilles de la jeune fille comme une corde à chaque tour plus courte. (Tf, 95 ; GW II, 286)

Quand le mystère de la conception et de la maladie plane au-dessus du couple et que la raison se voit obligée de défendre ses positions, le vocabulaire prend un caractère inquisitorial. N, qui " était bien un homme, et nullement un fou ", essaie "d'arracher un aveu à Tonka » (Tf, $100 ; G W$ II, 289). Il sait qu'il doit " se montrer tenace et se tenir à l'affût » (Tf, 102; GW II, 290), il s'emporte ou, au contraire, tente « de la prendre au piège en lui posant une question faussement anodine destinée à faire trébucher sa prudence » (Tf, 109; GW II, 295), et il finit par comprendre que même « torturée » elle n'avouerait pas (cf. Tf, 102 ; GW II, 290).

51 En se mettant ainsi en scène, la raison ici voisine, en effet, avec la torture. Cette raison n'est pas en mesure, pour revenir à la pensée de Musil, de répondre aux questions dont dépend la vie de notre âme, et en ce sens, on peut dire que la lumière qu'elle répand ne fait pas vivre, mais donne la mort : 
[...] le soleil semblait éclairer l'appartement à travers des vitres de plomb, illuminant et soulignant chaque objet d'un éclat mort. [...] Dans l'étrange lumière de la chambre, les objets ressemblaient maintenant à leurs propres momies. (Tf, 120 ; GW II, 302) pas vraiment au monde "éloigné de toute vérité»(Tf, 100; GW II, 289) qu'elle représente. Il peut avoir l'impression, pendant quelques instants, qu'il faudrait tout considérer autrement, selon d'autres critères. Il peut même aller jusqu'à se dire qu'il croit son amie, mais sans jamais oublier qu'il

ne la croyait en effet que dans la mesure où il se sentait incapable de continuer à se montrer sceptique et méchant envers elle, mais non jusqu’à répondre devant sa raison de toutes les conséquences que cela supposait. Ce fut cette réserve qui le sauva et l'empêcha de perdre pied. (Tf, 122 s.; GW II, 304)

$\mathrm{Si}$ « cette réserve » le sauve, elle coûte la vie à sa compagne. Tonka meurt à l'hôpital, elle n'aura été qu'un «mythe à demi avorté » (« ein halbgeborener Mythos») [trad. C.E. ; GW II, 303].

Les textes du recueil Trois femmes envisagent les rapports difficiles et parfois conflictuels entre le « ratioïde » et le «non ratioïde », entre, d'une part, la rationalité et l'univocité, et la mystique et la métaphore de l'autre. Quand on compare Tonka aux deux autres nouvelles ou à la pièce Les exaltés, écrite pour l'essentiel entre 1918 et 1920, ou encore aux essais datant de la même époque, on est saisi par un décalage : dans les autres textes littéraires, les héros masculins, en dignes précurseurs de l'homme sans qualités, non seulement captent des signaux émanant d'un autre monde mystérieux, mais quittent le domaine clôturé de la rationalité pour s'aventurer dans l'inconnu. Ainsi, on peut lire dans $L a$ Portugaise à propos du guerrier qu'est le seigneur von Ketten :

[...] commander est chose claire ; cette vie est claire comme le jour, solide comme un objet [« dingfest»], enfoncer un épieu dans le défaut d'une cuirasse est aussi simple que de montrer du doigt en disant : c'est ceci ou c'est cela. Ce qui est autre nous est aussi étranger que la lune. Le seigneur von Ketten aimait secrètement cet « autre ». (GW II, 259 [trad. C.E.])

Et Ketten ira vers cet « autre » tout comme le géologe Homo, qui dans l'etrange paysage montagneux du val Fersena tombe dans un état d'exaltation quasi mystique :

De quelque santé qu'Homo eût joui auparavant déjà, il se redressa alors comme un paralysé qui rejette soudain ses béquilles, se lève et marche. (Tf, 34 ; GW II, 248)

$\mathrm{N}$, lui, reste prisonnier de son intellect ${ }^{41}$, incapable de détourner vers Tonka ne seraitce qu'une part de l'énergie qu'il voue à son invention (cf. Tf, 122 ; GW II, 303), incapable donc de faire un pas vers la synthèse dont Musil prône les avantages dans ses essais. Le héros incarne une rationalité à la fois étriquée et tyrannique qui ne peut prétendre à une quelconque exemplarité et qui n'est pas un moyen propice à l'exploration du « non ratioïde ». Force est donc de constater qu'avec la nouvelle Tonka, Musil est en deçà du stade de conscience qu'il avait déjà atteint et dont témoigne son œuvre littéraire et théorique.

L'explication de ce décalage se trouve peut-être dans la biographie de l'auteur et dans un petit passage de la nouvelle. Quand Tonka est hospitalisée, $\mathrm{N}$ lui écrit des lettres, mais ne les poste pas. Elles

n'étaient d'ailleurs pas sa véritable pensée [«waren ja nicht mit Sicherheit seine Meinung »], plutôt le reflet d'un état dont il ne pouvait sortir qu'en écrivant. (Tf, 123 ; GW II, 304) 
Cette phrase traduit, selon Karl Eibl, la fiction de base («Basisfiktion ») de la nouvelle Tonka : un homme, assis à son bureau, essaie, par le biais d'un récit à la troisième personne, de mettre de l'ordre et de la continuité dans une période troublante de sa vie. Le travail de mémoire devient ici un travail de deuil et la nouvelle est le lieu où ce travail peut être entrepris ${ }^{42}$. Le héros fictif de Tonka, l'auteur des lettres non envoyées, se fond ainsi avec l'auteur de la nouvelle qui, et la phrase citée ci-dessus le suggère, se trouve lui aussi dans un état dont il ne peut sortir qu'en écrivant.

Il est évident que la nouvelle s'inspire de l'expérience vécue et que s'il y a un fil qui conduit de Herma Dietz à Tonka, il existe aussi des liens étroits entre Robert Musil et N. On a souvent insisté sur l'aspect autobiographique de ce texte. Ainsi, Rolf Schneider pense que dans Tonka Musil traite de sa propre histoire ${ }^{43}$. Tout aussi intéressantes que la correspondance entre la vie et l'écrit sont les modifications auxquelles l'auteur procède dans ses textes. Elles sont souvent la projection de désirs secrets, comme par exemple l'apparence physique de l'homme sans qualités. Musil, assez peu satisfait de sa petite taille $(1,64 \mathrm{~m})$, de sa calvitie et de sa forte transpiration, gratifie Ulrich, son alter ego, d'une taille élevée, d'une chevelure blonde et d'une peau à la sécheresse vaporeuse ${ }^{44}$. Dans Tonka, le besoin qu'éprouve l'auteur de « corriger la réalité » apparaît essentiellement dans deux phrases. Au moment où $\mathrm{N}$ s'interroge sur l'origine de la maladie dont est atteinte sa compagne, on peut lire qu' « à vues humaines, lui-même n'était pas malade » (Tf, 98 ; GW II, 288) et plus tard, quand Tonka est sur le point de s'éteindre, son ami se rassure : «Les médecins n'avaient jamais réussi à lui découvrir la moindre maladie » (Tf, 122; GW II, 303). C'est ce que dit la nouvelle. La vie réelle en avait décidé autrement.

Adolf Frisé a publié un formulaire (cf. Tb II, 1010-1013) comportant des renseignements anamnestiques que Musil a fournis à l'occasion d'une hospitalisation en 1916 et qui confirment ce que quelques notes dans son journal (cf. $\mathrm{Tb} \mathrm{I}, 16,697)$ laissent entendre : il avait lui-même contracté la syphilis, fort probablement avant de connaître Herma Dietz. Etant donné que le traitement avant l'apparition des antibiotiques (embrocations et applications d'iode) ne permettait qu'une rémission de la maladie, mais sans guérison véritable, on peut penser que c'est Musil qui a contaminé son amie.

L'histoire vécue avec Herma Dietz pèse apparemment si lourd dans la conscience de Musil qu'il tente, environ quinze ans après les événements, d'en finir par l'écriture. Dans cette perspective, la nouvelle est la manifestation littéraire d'un souvenir refoulé de l'auteur. L'expérience culpabilisante se voit ainsi projetée dans une problématique de l'époque, à savoir l'opposition entre le «ratioïde » et le "non ratioïde», et la contamination de la compagne devient alors un phénomène mystique. Dans cette constellation, Musil attribue sa propre culpabilité à la raison, qu'il présente sous un jour bien moins favorable que dans les autres textes. La raison incarnée par $\mathrm{N}$ ressemble alors beaucoup plus à la raison despotique dont parle Foucault qu'à celle dont Musil, dans d'autres circonstances, ne cesse de louer les vertus. Sous le poids de la mémoire, il déforme sa pensée, et même il la trahit. 


\section{Textes de Robert Musil (avec les abréviations utilisées) :}

GW I : Der Mann ohne Eigenschaften, hrsg. von Adolf Frisé, Rowohlt, Reinbek bei Hamburg, 1978. GW II : Prosa und Stücke. Kleine Prosa, Aphorismen, Autobiographisches, Essays und Reden. Kritik, hrsg. von Adolf Frisé, Rowohlt, Reinbek bei Hamburg, 1978.

Tb I : Tagebücher, hrsg. von Adolf Frisé, Rowohlt, Reinbek bei Hamburg, 1976.

Tb II : Tagebücher. Anmerkungen, Anhang, Register, hrsg. von Adolf Frisé, Rowohlt, Reinbek bei Hamburg, 1976.

B I : Briefe 1901-1942, hrsg. von Adolf Frisé (unter Mithilfe von Murray G. Hall), Rowohlt, Reinbek bei Hamburg, 1981.

L'Hsq 1 et 2 : L'Homme sans qualités, tomes 1 et 2, traduits de l'allemand par Philippe Jaccottet, Editions du Seuil, Paris, 1995.

Tf : Trois femmes suivi de Noces, traduit de l'allemand par Philippe Jaccottet, Editions de Seuil, Paris, 1962.

E : Essais, conférences, critique, aphorismes et réflexions, textes choisis, traduits et présentés par Philippe Jaccottet, Editions du seuil, Paris, 1984.

Musil Robert, « Testament II » (trad. par Philippe Jaccottet), in Roth Marie-Louise, Olmi Roberto (ed.), Robert Musil, L’Herne, n41, Paris, 1981, p. 37-40.

\section{Études sur Robert Musil et ouvrages généraux}

Bouveresse Jacques, «Introduction », in Bouveresse Jacques, La voix de l'âme et les chemins de l'esprit. Dix études sur Robert Musil, Éditions du Seuil, [Paris], 2001, p. 11-84.

Bouveresse Jacques, « Robert Musil, la philosophie de la vie et les illusions de l'Action parallèle », in Bouveresse Jacques, La voix de l'âme et les chemins de l'esprit. Dix études sur Robert Musil, Editions du Seuil, [Paris], 2001, p. 189-226.

Broch Hermann, « Robert Musil und das Exil », in Kommentierte Werkausgabe, hrsg. von Paul Michael Lützeler, Band 9/1, Suhrkamp, Frankfurt am Main, 1975, p. 96-97.

Corino Karl, Robert Musil. Leben und Werk in Bildern und Texten, Rowohlt, Reinbek bei Hamburg, 1986.

Eibl Karl, Robert Musil. Drei Frauen. Text, Materialien, Kommentar, Carl Hanser, München-Wien, 1978. Foucault Michel, Histoire de la folie à l'âge classique, Gallimard, [Paris], 1972.

Homann Renate, «Literatur und Erkenntnis : Robert Musils Erzählung 〈Tonka〉 », in Deutsche Vierteljahrsschrift für Literaturwissenschaft und Geistesgeschichte, Stuttgart, 1985, p. 497-518.

Kiesel Helmuth, "Aufklärung und neuer Irrationalismus in der Weimarer Republik », in Schmidt Jochen (Hrsg.), Aufklärung und Gegenaufklärung in der europäischen Literatur, Philosophie und Politik von der Antike bis zur Gegenwart, Wissenschaftliche Buchgesellschaft, Darmstadt, 1989, p. 497-521. Revel Judith, Le vocabulaire de Foucault, Ellipses, Paris, 2002.

Schneider Günther, Untersuchungen zum dramatischen Werk Robert Musils, Peter Lang, BernFrankfurt/M, 1973 ( = Europäische Hochschulschriften, Bd. 81).

Schneider Rolf, Die problematisierte Wirklichkeit. Leben und Werk Robert Musils. Versuch einer Interpretation, Verlag Volk und Welt, Berlin 1975.

Sokel Walter, Kleists 'Marquise von O.', Kierkegaards 'Abraham' und Musils 'Tonka' : Drei Stufen des 
Absurden in seiner Beziehung zum Glauben, in Dinklage Karl e.a. (Hrsg.), Robert Musil. Studien zu seinem Werk, Rowohlt, Reinbek bei Hamburg, 1970, p. 57-70.

Weber Max, «Wissenschaft als Beruf », in Gesamtausgabe, hrsg. von Horst Baier, M. Rainer Lepsius e.a., Band 17, J.C.B. Mohr (Paul Siebeck), Tübingen 1992, p. 71-111.

Willemsen Roger, Robert Musil. Vom intellektuellen Eros, Piper, München, 1985.

\section{NOTES}

1. Robert Musil, «Testament II (trad. par Philippe Jaccottet), in Marie-Louise Roth, Roberto Olmi (ed.), Robert Musil, L’Herne, n41, Paris, 1981, p. 38 s. (GW II, 954).

2. Cf. Karl Corino, Robert Musil. Leben und Werk in Bildern und Texten, Rowohlt, Reinbek bei Hamburg, 1986, p. 94.

3. Ibid., p. 84.

4. Ibid.., p. 118.

5. Ibid., p. 12 et 136.

6. « [...] weil ich dich noch lange nicht für mir gleichwertig halte [.] » (Tb I, 15).

7. La note que Musil inscrit le 2 juin 1902 dans son journal commence par les mots : « Ein Thema für den Herrn Schriftsteller : [...] » (Tb I, 23).

8. Voir note 1

9. Cf. Günther Schneider, Untersuchungen zum dramatischen Werk Robert Musils, Peter Lang, BernFrankfurt/M, 1973 (= Europäische Hochschulschriften, Bd. 81), p. 2.

10. Hermann Broch, «Robert Musil und das Exil », in Kommentierte Werkausgabe, hrsg. von Paul Michael Lützeler, Band 9/1, Suhrkamp, Frankfurt am Main, 1975, p. 96 (trad. C.E.).

11. Cf. Roger Willemsen, Robert Musil. Vom intellektuellen Eros, Piper, München, 1985, p. 60.

12. Jacques Bouveresse, "Introduction", in Jacques Bouveresse, La Voix de l'âme et les chemins de l'esprit. Dix études sur Robert Musil, Editions du Seuil, [Paris], 2001, p. 50.

13. Max Weber, "Wissenschaft als Beruf ", in Gesamtausgabe, hrsg. von Horst Baier, M. Rainer Lepsius e.a., Band 17, J.C.B. Mohr (Paul Siebeck), Tübingen 1992, p. 86 : « Der wissenschaftliche Fortschritt ist ein Bruchteil, und zwar der wichtigste Bruchteil jenes Intellektualisierungsprozesses, [...] zu dem heute üblicherweise in so außerordentlich negativer Art Stellung genommen wird. »

14. Ibid., p. 92 : «Erlösung von dem Rationalismus und Intellektualismus der Wissenschaft ist die Grundvoraussetzung des Lebens in der Gemeinschaft mit dem Göttlichen : dies oder etwas dem Sinn nach Gleiches ist eine der Grundparolen, die man aus allem Empfinden unserer religiös gestimmten oder nach religiösem Erlebnis strebenden Jugend heraushört. Und nicht nur [nach dem] religiöse[n], nein [nach dem] Erlebnis überhaupt. »

15. Cf. Helmuth Kiesel, «Aufklärung und neuer Irrationalismus in der Weimarer Republik », in Jochen Schmidt (Hrsg.), Aufklärung und Gegenaufklärung in der europäischen Literatur, Philosophie und Politik von der Antike bis zur Gegenwart, Wissenschaftliche Buchgesellschaft, Darmstadt, 1989, p. 509.

16. Ibid., p. 503 s.

17. «[...] [daß die Lessingsche Aufklärung] heute geistig veraltet ist und einem blutvolleren, tieferen, tragischeren Lebensbegriff Platz gemacht hat ». Cité d'après Kiesel, " Aufklärung... ", p. 504.

18. Jacques Bouveresse, par exemple, pense que «L'Homme sans qualités peut et doit être compris, pour une part essentielle, comme une critique implicite de la philosophie de la vie ». Jacques Bouveresse, "Robert Musil, la philosophie de la vie et les illusions de l'Action parallèle ", in Bouveresse, La Voix de l'âme..., p. 190. 
19. L'essentiel de ces réflexions est tiré d'un essai de 1922 qui s'intitule : L'Europe désemparée ou petit voyage du coq à l'âne (Das hilflose Europa oder Reise vom Hundertsten ins Tausendste).

20. Voici la phrase entière : "Elle était comme un flocon de neige qui tomberait en plein été ». Philippe Jaccottet, qui a traduit l'œuvre de Musil en français et dont les mérites sont notoires, a, semble-t-il, voulu atténuer la force de la métaphore. Il compare (« comme ») là où Musil identifie, il emploie le conditionnel où Musil a opté pour le participe présent. En outre, Musil, en utilisant l'adverbe "allein", accentue l'aspect d'isolement: "Eine mitten an einem Sommertag allein niederfallende Schneeflocke war sie » (GW II, 304). L'image apparaît une deuxième fois dans la nouvelle. Les deux versions sont les suivantes : «Ist [...] solcher Mensch, solche mitten in einem Sommertag ganz allein niederfallende Schneeflocke Wirklichkeit oder Einbildung [...] ?» (GW II, 280) - « [...] un être pareil à un flocon de neige égaré en plein été, est-il réalité ou rêve [...] ? (Tf, $85 \mathrm{s.})$

21. Comme certains critiques l'ont fait, nous appelons « $N$ » l'ami de Tonka, qui n'a pas de nom dans la nouvelle.

22. "Auch war er ja nach menschlichem Ermessen nicht krank, und es verstrickte ihn also entweder ein mystischer Zusammenhang mit Tonka oder sie hatte gemeine irdische Schuld auf sich geladen. » (GW II, 288).

23. "Man fühlt, daß da die Begriffe an eine Grenze kommen, wo sie keinen Halt mehr finden. » (GW II, 280)

24. Dans un brouillon de lettre de 1907, on lit: «Aber Gleichnisse sind wie Musik am Abend, die irgendwoher kommt, aus irgendeinem einsamen, hinter Büschen verborgenen Hause, darinnen es zum Träumen ist. Man weiß nicht, wo es ist $u$. welche Träume es birgt. Und man wird es nicht wissen, denn gleich wird mit dem Abend die Musik verlöschen. » (Br I, 33 s.)

Pour décrire l'effet créé par le Gleichnis, Musil emploie ici les mêmes éléments (Musik, Abend, einsames Haus, verlöschende Musik) que le narrateur de la nouvelle, quand il se souvient de Tonka.

25. Cf. par ex. les deux essais Le spirituel, le modernisme et la métaphysique (Das Geistliche, der Modernismus und die Metaphysik) de 1912 et La connaissance chez l'écrivain: esquisse (Skizze der Erkenntnis des Dichters) de 1918.

26. Cf. Michel Foucault, Histoire de la folie à l'âge classique, Gallimard, [Paris], 1972, p. 22.

27. Ibid., p. 26.

28. Ibid., p. 41.

29. Ibid., p. 37.

30. Ibid., p. 115.

31. Ibid., p. 120.

32. Cf. ibid., p. 125.

33. Ibid., p. 48.

34. Judith Revel, Le vocabulaire de Foucault, Ellipses, Paris, 2002, p. 34.

35. Foucault, Histoire de la folie..., p. 637.

36. Ibid., 638.

37. Ibid., p. 63.

38. « H. [= Herma/Tonka] [...] Unbeholfen wie aus einem altdeutschen Bild». (Tb I, 100).

39. Renate Homann, «Literatur und Erkenntnis : Robert Musils Erzählung Tonka», in Deutsche Vierteljahrsschrift für Literaturwissenschaft und Geistesgeschichte, Stuttgart, 1985, p. 507.

40. Cf. Karl Eibl, Robert Musil. Drei Frauen. Text, Materialien, Kommentar, Carl Hanser, MünchenWien, 1978, p. 152.

41. Cf. Walter Sokel, Kleists 'Marquise von O.', Kierkegaards 'Abraham' und Musils 'Tonka' : Drei Stufen des Absurden in seiner Beziehung zum Glauben, in Karl Dinklage e.a. (Hrsg.), Robert Musil. Studien zu seinem Werk, Rowohlt, Reinbek beiHamburg, 1970, p. 64.

42. Cf. Karl Eibl, Robert Musil..., p. 139 et 155. 
43. Cf. Rolf Schneider, Die problematisierte Wirklichkeit. Leben und Werk Robert Musils. Versuch einer Interpretation, Verlag Volk und Welt, Berlin 1975, p. 66 et 69.

44. Cf. Corino, Robert Musil..., p. 490.

\section{RÉSUMÉS}

La situation chaotique dans laquelle se trouve le domaine de l'esprit au début du xx $\mathrm{e}^{\mathrm{e}}$ siècle est due pour Robert Musil au fait que les deux aspects de l'esprit humain (raison - sentiment, rationalité - mystique) ne se sont jamais unis dans une synthèse.Depuis la pièce Die Schwärmer, les protagonistes masculins tentent tous de réaliser une telle synthèse, à l'exception de l'ami dans la nouvelle Tonka. Dans ce texte, la mémoire que l'auteur a conservée d'une période sombre de sa vie interfère avec sa pensée et garde le héros de la nouvelle prisonnier d'une rationalité stérile.

Als entscheidend für die chaotische geistige Situation seiner Zeit wertet Robert Musil den Umstand, dass sich die beiden Perspektiven des menschlichen Geistes (Verstand - Gefühl, Ratio Mystik) nie zu einer Synthese vereinigt haben. Um eine solche bemühen sich seit dem Schauspiel Die Schwärmer alle männlichen Hauptfiguren Musils, mit Ausnahme des Freundes in der Novelle Tonka.In diesem Text interferiert die Erinnerung des Autors an einen dunklen Abschnitt seines Lebens mit seinem Denken und lässt den Protagonisten der Novelle nicht über einen unfruchtbaren Rationalismus hinausgelangen.

\section{AUTEUR}

\section{CLAUS ERHART}

Université de Nice 